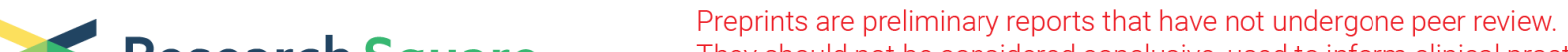 Research Square They should not be considered conclusive, used to inform clinical practice, or referenced by the media as validated information.
}

\section{External Assessment of the EUROMACS Right-Sided Heart Failure Risk Score}

Hirak Shah

University of Minnesota

Thomas Murray

University of Minnesota

Jessica Schultz

University of Minnesota

Ranjit John

University of Minnesota

Cindy Martin

University of Minnesota

Thenappan Thenappan

University of Minnesota

Rebecca Cogswell ( $\square$ cogsw014@umn.edu)

University of Minnesota

\section{Research Article}

Keywords: EUROMACS Right-Sided Heart Failure Risk Score, assessment, RVF, LVAD

Posted Date: March 3rd, 2021

DOI: https://doi.org/10.21203/rs.3.rs-244191/v1

License: (c) (i) This work is licensed under a Creative Commons Attribution 4.0 International License. Read Full License

Version of Record: A version of this preprint was published at The Journal of Heart and Lung Transplantation on April 1st, 2019. See the published version at https://doi.org/10.1016/j.healun.2019.01.901. 


\section{Abstract}

The EUROMACS Right-Sided Heart Failure Risk Score was developed to predict right ventricular failure (RVF) after left ventricular assist device (LVAD) placement. The predictive ability of the EUROMACS score has not been tested in other cohorts. We performed a single center analysis of a continuous-flow (CF) LVAD cohort $(n=254)$ where we calculated EUROMACS risk scores and assessed for right ventricular heart failure after LVAD implantation. Thirty-nine percent of patients (100/254) had post-operative RVF, of which 9\% (23/254) required prolonged inotropic support and 5\% (12/254) required RVAD placement. For patients who developed RVF after LVAD implantation, there was a 45\% increase in the hazards of death on LVAD support (HR 1.45, $95 \% \mathrm{Cl} 0.98-2.2, \mathrm{p}=0.066)$. Two variables in the EUROMACS score (Hemoglobin and Right Atrial Pressure to Pulmonary Capillary Wedge Pressure ratio) were not predictive of RVF in our cohort. Overall, the EUROMACS score had poor external discrimination in our cohort with area under the curve of $58 \%$ (95\% $\mathrm{Cl} 52-66 \%)$. Further work is necessary to enhance our ability to predict RVF after LVAD implantation.

\section{Introduction}

Right ventricular (RV) failure remains common after left ventricular assist device placement (LVAD) even in contemporary continuous flow era ${ }^{1}$, and remains a leading cause of morbidity and mortality after LVAD placement ${ }^{2,3}$. A number of definitions of early right ventricular failure after LVAD exist. All include unplanned right ventricular assist device (RVAD) placement after LVAD implantation, however definitions vary by length and use of pulmonary vasodilators or intravenous inotropes ${ }^{1,4,5}$. Several prediction tools have been developed to try to capture risk of postoperative RV failure with pre-operative variables, however the performance of these models has been variable to poor on external validation ${ }^{3,6}$. One of the more recently published risk models was developed from the EUROMACS database ${ }^{7}$. This analysis included 2,988 patients implanted with LVADs in Europe in which 433 patients (21.7\%) developed right ventricular failure. After performing logistic regression, a combination of five variables were found to be highly predictive of right sided heart failure. These variables include right atrial/pulmonary capillary wedge pressure $>0.54$, hemoglobin $<10 \mathrm{~g} / \mathrm{dL}$, use of multiple intravenous inotropes prior to LVAD implantation, INTERMACS Class 1-3, and severe right ventricular dysfunction on echocardiography. The C-Statistic for this risk score was 0.7 , which was higher than other risk scores that have been published. The performance of this model outside the EUROMACs cohort has not been tested. Therefore, the purpose of this study was to assess the performance of the EUROMACs score in a large, external continuous flow LVAD dataset and assess for other univariate predictors of RV failure.

\section{Methods}

\section{Cohort and Inclusion Criteria}

The institutional review board of the University of Minnesota Medical Center approved this study.

The requirement of informed consent for the study is waived by the institutional review board of the University of Minnesota.

All methods were carried out in accordance with relevant guidelines and regulations.

At the time of this analysis, the larger continuous-flow LVAD cohort consisted of 451 patients implanted between 2007-2017. Of these, 254 patients met the following inclusion criteria 1) first time, continuous-flow LVAD implantation 2) complete pre-operative variables to complete EUORMACS score calculation, and 3) complete post-operative data to determine date of inotrope wean, pulmonary vasodilator wean and RVAD use. 
The following demographic and clinical covariate data are available in the University of Minnesota LVAD database, which is updated through data extraction and manual chart review: age, gender, body mass index (BMI), serum creatinine, albumin, Interagency Registry for Mechanical Circulatory Support (INTERMACS) profile, pre-operative hemodynamics, bridge to transplant status, cardiomyopathy type, presence of diabetes, and NT pro b-type natriuretic peptide (NT-proBNP). Vital status was obtained from chart review. The date of the last clinic visit was recorded for patients who were still alive at the end of follow up. For cardiac transplantation, the date of cardiac transplant was obtained from the electronic medical record and confirmed with an operative report.

\section{Primary predictor and outcome}

The primary predictor for this analysis was follow up EUROMACS RV score, which was calculated by totaling points for the following pre-LVAD clinical variables. RA/PCWP > 0.54: 2 points, hemoglobin $\leq 10 \mathrm{~g} / \mathrm{dL}: 1$ point, multiple inotropes: 2.5 points, INTERMACS 1-3: 2 points, and severe RV dysfunction: 2 points. The primary outcome was early severe post-operative right ventricular failure defined as need for short/long-term mechanical right-sided circulatory support within 30 days of LVAD implantation, continuous inotropic support greater than or equal to 14 days, or need for pulmonary vasodilators for greater than 48 hours.

\section{Statistical Methods:}

All statistical analyses were performed using STATA 16 (College Station, Texas). A p-value of $<0.05$ was considered statistically significant. Baseline characteristics between cohort subjects who developed early RVF and those who did not develop early RVF were compared with normally distributed continuous variables were compared with t-tests and Wilcoxon rank sum tests for non-normally distributed continuous variables. Categorical variables were compared with Pearson chi-squared tests or Fishers exact test, where appropriate. In order to determine the relationship between baseline co-variates and follow up pulmonary capillary wedge pressure, multivariable linear regression analyses were performed.

In order to assess EUROMACS model discrimination, a receiver operator curve was generated with EUROMACS score as the predictor and post LVAD RVF as the outcome. The EUROMACS score was also assessed as a predictor of RVF using logistic regression. In order to determine the relationship between other pre-operative variables and RVF, logistic regression was performed. The relationship between RVF (or RVAD use alone) and subsequent LVAD mortality was assessed with cox regression.

\section{Results}

The mean age of the cohort was 59 years of age. Eighty two percent were male, $80 \%$ were Caucasian, and $51 \%$ were designated as bridge to transplantation. The majority of patients (52\%) were INTERMACS profile $2-3$. A total of 100 patients (39\%) were diagnosed with early right ventricular failure and 154 patients $(61 \%)$ did not have post-operative right heart failure. There were no significant differences in age, sex, race, baseline atrial fibrillation, cardiomyopathy type, diabetes, chronic obstructive pulmonary disease, LVAD surgical strategy, INTERMACS profile, pulmonary artery systolic pressure or cardiac index between both groups (Table 1).

\section{Outcomes of Right Heart Failure}

Of the 100 patients who met RV failure criteria, 12 patients required RVAD and 23 patients required inotropes $>14$ days. Patients who required pulmonary vasodilators $>48$ hours comprised the vast majority $(n=65)$ of patients who were defined as having post-operative RVF. Early right-sided heart failure was associated with increased mortality 
(unadjusted HR $1.45 ; 95 \% \mathrm{Cl} 0.98-2.16, \mathrm{p}=0.066$ ). Use of RVAD after LVAD surgery was the primary driver of increased mortality (HR 4.56; 95\% Cl 2.28-9.11, p < 0.001, Fig. 1).

\section{Risk Factor for Early Postoperative Right Heart Failure}

We tested 66 distinct risk factors for early post-operative right heart failure. An additional nine factors were calculated from these 66 variables (Table 2a-c). Of the individual variables tested, a higher pulmonary arterial pressure was protective against RV failure as was a higher pulmonary arterial pulse pressure. Higher creatinine, lower albumin, and higher total bilirubin were all associated with increased risk of RV failure after LVAD. Pre-operative ventilator support, multiple inotropes, extra corporal membrane oxygenator support, and non-elective IABP were also associated with higher risk of RVF. Of note, hemodynamic parameters such as right atrial pressure, right atrial/pulmonary capillary wedge pressure, pulmonary artery pulsatility index, pulmonary artery compliance, and pulmonary artery elastance were not associated with increased risk of right sided heart failure in this dataset.

\section{Validation of the EUROMACS Score}

An elevated EUROMACS score was associated with increased risk of RVF (OR 6.88; 95\% $\mathrm{Cl} 2.31$ to 20.45, $\mathrm{p}=0.001$ ). The EUROMACS score had an area under the curve of 59.3\%, (95 \% Cl 52.2-66.3 \%) (Fig. 2).

\section{Discussion}

This is the first study to test the performance of the EUROMACs score to predict right ventricular failure after CF-LVAD implantation in an external dataset to the development cohort. We found that right ventricular failure was associated with mortality after CF-LVAD. This was mainly driven by right ventricular failure defined by need for RVAD and/or prolonged inotropes. Importantly, we found that the EUROMACS score had poor discrimination in predicting RV failure. Many of the variables used in the EUROMACs score were not associated with RV failure in our cohort including hemoglobin and RA/PCWP ratio.

Right heart failure is one of the most common causes of early morbidity and mortality after CF-LVAD implantation. Predicting RV failure is important as planned institution of RVAD during LVAD surgery has been associated with improved outcomes compared to delayed RVAD implantation 8,9 , and a direct to transplant strategy can be employed in eligible patients to avoid this severe complication. The present study again demonstrates the difficulty in predicting RV failure after LVAD, even with the most contemporary risk scores.

There are many reasons why our results may have differed from the original EUROMACs analysis. In our cohort, the incidence of the RV failure occurred in 39\% patients undergoing CF-LVAD implantation. The majority of the patients (65\%) met this definition due to prolonged pulmonary vasodilator use, while the EUROMACs data only had $1 \%$ of patients with prolonged pulmonary vasodilator use. When restricting the definition to inotropes $>14$ days and/or need for RVAD, only $14 \%$ of our cohort met the definition of RV failure. In our cohort, RV failure defined by prolonged vasodilator use was not associated with increased mortality, which questions the clinical significance of this part of the RV failure definition. In addition, the EUROMACs cohort had less destination therapy CF-LVAD implantations (14\% vs. $49 \%)$.

There have been several previous studies designed to predict those patients at high likelihood of developing RV failure following LVAD implantation. Many of these include hemodynamic variables such as right atrial pressure, pulmonary artery pulsatility index, and right atrial pressure: pulmonary capillary wedge pressure ratio ${ }^{10,11}$. Other studies have shown that certain echocardiographic features of RV dysfunction such as TAPSE and semi-quantitative RV function 
are predictive of RV failure after LVAD ${ }^{12,13}$. In our cohort, none of the previously mentioned hemodynamic variables were predictive of RV failure. Similar to previous analyses, higher creatinine, lower albumin, and higher total bilirubin were all associated with increased risk of RV failure after LVAD. Pre-operative ventilator support, multiple inotropes, extra corporal membrane oxygenation, and non-elective IABP were also associated with higher risk of RVF in this cohort. All of the associated variables are direct or indirect markers of patient acuity, and may suggest longer standing heart failure is a risk factor for post-operative RV failure.

There are many reasons why published risk scores may perform poorly in validation cohorts. First, RV failure after LVAD does not have a universal definition ${ }^{14}$. For example, the widely used INTERMACS criteria for RV failure does not include pulmonary vasodilators as definition for RVF. With regard to the hemodynamic variables, these can change dramatically over a 24 hour period as patient are managed with diuretics, inotropes and temporary support. Lastly, insults that occur to the RV in the operating room, such as RV ischemia from hypotension, prolonged cardiopulmonary bypass time, surgical positioning of the inflow cannula, fluid resuscitation and blood transfusions are not predictable ahead of time ${ }^{15}$. These intraoperative conditions can "unmask" underlying RV failure that might not have been predicted with traditional risk scores using clinical, hemodynamic, and echocardiographic data.

This study has several limitations. First, this was a single-center observational analysis. Second, our sample size is smaller than the EUROMACs cohort, which could limit the power of our study to determine variables that are associated with severe RV failure.

\section{Conclusion}

The EUROMACS Right-Sided Heart Failure Risk Score had poor external discrimination in this LVAD dataset. These findings further emphasize the difficulty of predicting RVF after LVAD placement. Further work is required to enhance our understanding of post-LVAD RVF.

\section{Abbreviations}

BNP: brain natriuretic peptide

BMl: body mass index

COPD: chronic obstructive pulmonary disease

EUROMACS: European Registry for Patients with Mechanical Circulatory Support

INR: international normalizing ratio

INTERMACS: Interagency Registry for Mechanical Circulatory Support

LVAD: left ventricular assist device

RVF : right ventricular failure

\section{Declarations}

Funding: University of Minnesota Departmental Funds

Conflicts of Interest:

Page 5/14 
Ranjit John, MD

Abbott Lab: consultant, research grants

Medtronic: consultant, research grants

Rebecca Cogswell, MD

Abbott Lab: consultant, speaker's bureau

Medtronic: consultant, speaker's bureau, husband's employment, Heart Failure Advisory Board

Thomas Murray, PhD

Medtronic: Medtronic Biostatistics Faculty Fellow

\section{References}

1. Mehra, M. R. et al. A Fully Magnetically Levitated Left Ventricular Assist Device - Final Report. N Engl J Med. 380, 1618-1627 https://doi.org/10.1056/NEJMoa1900486 (2019).

2. Dang, N. C. et al. Right heart failure after left ventricular assist device implantation in patients with chronic congestive heart failure. J Heart Lung Transplant. 25, 1-6 https://doi.org/10.1016/j.healun.2005.07.008 (2006).

3. Kalogeropoulos, A. P. et al. Validation of clinical scores for right ventricular failure prediction after implantation of continuous-flow left ventricular assist devices. J Heart Lung Transplant. 34, 1595-1603 https://doi.org/10.1016/j.healun.2015.05.005 (2015).

4. Kormos, R. L. et al. Right ventricular failure in patients with the HeartMate II continuous-flow left ventricular assist device: incidence, risk factors, and effect on outcomes. The Journal of thoracic and cardiovascular surgery. 139, 1316-1324 (2010).

5. Kormos, R. L. et al. The Society of Thoracic Surgeons Intermacs database annual report: Evolving indications, outcomes, and scientific partnerships. The Journal of Heart and Lung Transplantation 38, 114-126(2019).

6. Pettinari, M. et al. Are right ventricular risk scores useful? European journal of cardio-thoracic surgery. 42, 621-626 (2012).

7. Soliman, O. I. et al. Derivation and validation of a novel right-sided heart failure model after implantation of continuous flow left ventricular assist devices: the EUROMACS (European Registry for Patients with Mechanical Circulatory Support) right-sided heart failure risk score. Circulation. 137, 891-906 (2018).

8. Takeda, K. et al. Outcome of unplanned right ventricular assist device support for severe right heart failure after implantable left ventricular assist device insertion. The Journal of Heart and Lung Transplantation. 33, 141-148 (2014).

9. Fitzpatrick, I. I. I. J. R. et al. Early planned institution of biventricular mechanical circulatory support results in improved outcomes compared with delayed conversion of a left ventricular assist device to a biventricular assist device. The Journal of thoracic and cardiovascular surgery. 137, 971-977 (2009).

10. Morine, K. J. et al. Pulmonary artery pulsatility index is associated with right ventricular failure after left ventricular assist device surgery. Journal of cardiac failure. 22, 110-116 (2016).

11. Drakos, S. G. et al. Risk factors predictive of right ventricular failure after left ventricular assist device implantation. The American journal of cardiology. 105, 1030-1035 (2010). 
12. Puwanant, S. et al. Tricuspid annular motion as a predictor of severe right ventricular failure after left ventricular assist device implantation. The Journal of heart and lung transplantation. 27, 1102-1107 (2008).

13. Fitzpatrick, I. I. I. J. R. et al. Risk score derived from pre-operative data analysis predicts the need for biventricular mechanical circulatory support. The Journal of Heart and Lung Transplantation. 27, 1286-1292 (2008).

14. Lampert, B. C. \& Teuteberg, J. J. Right ventricular failure after left ventricular assist devices. The Journal of Heart and Lung Transplantation. 34, 1123-1130 (2015).

15. Rich, J. D. The inherent fallacy of predicting $r$ failure following left ventricular assist device implantation. Journal of cardiac failure. $25,629-630$ (2019).

\section{Tables}

Table 1: Baseline Characteristics of the Cohort by Presence or Absence of Early Right Heart Failure by the 


\begin{tabular}{|c|c|c|c|}
\hline Variable & $\begin{array}{c}\text { No Early RHF } \\
\qquad(\mathrm{N}=154)\end{array}$ & $\begin{array}{l}\text { Early RHF } \\
(\mathrm{N}=100)\end{array}$ & P-value \\
\hline Age at LVAD Implantation & $62(54-69)[0]$ & $62(49.8-68.2)[0]$ & 0.3548 \\
\hline Male & 127 (82.5\%) [0] & $81(81 \%)[0]$ & 0.8677 \\
\hline Race White & $121(85.2 \%)$ & $83(88.3 \%)$ & 0.5588 \\
\hline Race Black & $12(8.5 \%)$ & $8(8.5 \%)$ & \\
\hline Race Other & $9(6.3 \%)$ & $3(3.2 \%)$ & \\
\hline Race Missing & 12 & 6 & \\
\hline Baseline Atrial Fibrillation & $68(44.7 \%)[2]$ & $47(47.5 \%)[1]$ & 0.6987 \\
\hline Ischemic Cardiomyopathy & $78(50.6 \%)[0]$ & $56(56 \%)[0]$ & 0.4414 \\
\hline Diabetes & 75 (49.3\%) [2] & 47 (47.5\%) [1] & 0.7971 \\
\hline Chronic Obstructive Pulmonary Disease & 35 (23\%) [2] & $25(25.3 \%)[1]$ & 0.7623 \\
\hline Bridge to Transplant & 79 (51.3\%) [0] & $50(50 \%)[0]$ & 0.8980 \\
\hline INTERMACS 1 & $14(9.1 \%)$ & $16(16 \%)$ & 0.1398 \\
\hline INTERMACS 2-3 & $78(50.6 \%)$ & $53(53 \%)$ & \\
\hline INTERMACS 4-7 & $62(40.3 \%)$ & $31(31 \%)$ & \\
\hline Body Mass Index $\left(\mathrm{kg} / \mathrm{m}^{2}\right)$ & $28(25-32.4)[6]$ & $29.3(26.1-33.3)$ [6] & 0.4767 \\
\hline Creatinine $(\mathrm{mg} / \mathrm{dL})$ & $1.1(0.9-1.6)[2]$ & $1.3(1.1-1.8)[1]$ & 0.0195 \\
\hline Albumin $(\mathrm{g} / \mathrm{dL})$ & $3.4(3-3.8)[2]$ & $3.3(2.9-3.5)[1]$ & 0.2103 \\
\hline Right Atrial Pressure (mmHg) & $10(7-15)[19]$ & $14(10-18)[19]$ & 0.0257 \\
\hline Pulmonary artery systolic pressure $(\mathrm{mmHg})$ & $50(40-60)[19]$ & $50(43-62)[19]$ & 0.5596 \\
\hline Pulmonary artery diastolic pressure $(\mathrm{mmHg})$ & $23(18-30)[19]$ & $26(21-32)[19]$ & 0.1473 \\
\hline Cardiac Index $\left(\mathrm{L} / \mathrm{min} / \mathrm{m}^{2}\right)$ & $1.9(1.6-2.3)[14]$ & $1.9(1.5-2.3)[15]$ & 0.9670 \\
\hline Pulmonary arterial compliance $\left(\mathrm{mL} \cdot \mathrm{mmHg}^{-1}\right)$ & $1.9(1.4-2.9)[23]$ & $1.8(1.3-2.4)[21]$ & 0.1802 \\
\hline Pulmonary artery pressure index & $2.4(1.6-4)[19]$ & $1.9(1.3-3.1)[19]$ & 0.0231 \\
\hline Right ventricular stroke work index ( $\mathrm{g} / \mathrm{m} / \mathrm{beat} / \mathrm{m}^{2}$ ) & $13.9(11-17)[23]$ & $13.4(10-17.9)[21]$ & 0.6542 \\
\hline
\end{tabular}

Legend: Continuous variables are described with median (IQR) [N Missing]. Binary variables are described with $\mathrm{N}(\%)$ [N Missing]

Table 2a: Odds-Ratio of each standardized continuous risk factor for early right heart failure based on univariate logistic regression models 


\begin{tabular}{|c|c|c|}
\hline Predictor & Odds-Ratio (95\% CI) & p-value \\
\hline Age (years) & $0.53(0.19,1.47)$ & $\mathrm{p}=0.221$ \\
\hline Body Mass Index $(\mathrm{kg} / \mathrm{m} 2)$ & $1.19(0.38,3.73)$ & $\mathrm{p}=0.761$ \\
\hline $\log$ NT-proBNP $(\mathrm{pg} / \mathrm{mL})$ & $1.06(0.36,3.12)$ & $\mathrm{p}=0.910$ \\
\hline Glomerular Filtration Rate $(\mathrm{mL} / \mathrm{min})$ & $0.18(0.05,0.66)$ & $\mathrm{p}=0.010^{*}$ \\
\hline Hemoglobin (g/dL) & $0.51(0.15,1.81)$ & $\mathrm{p}=0.300$ \\
\hline International Normalized Ratio & $2.77(1.28,6.00)$ & $\mathrm{p}=0.010^{*}$ \\
\hline Platelets & $0.79(0.24,2.66)$ & $p=0.707$ \\
\hline Creatinine $(\mathrm{mg} / \mathrm{dL})$ & $3.71(1.63,8.46)$ & $\mathrm{p}=0.002^{*}$ \\
\hline C-Reactive Protein & $2.49(1.09,5.72)$ & $\mathrm{p}=0.031 *$ \\
\hline Prealbumin $(\mathrm{mg} / \mathrm{L})$ & $0.21(0.05,0.85)$ & $\mathrm{p}=0.029 *$ \\
\hline Sodium & $1.33(0.41,4.30)$ & $\mathrm{p}=0.638$ \\
\hline $\operatorname{Albumin}(\mathrm{mg} / \mathrm{dL})$ & $0.22(0.07,0.69)$ & $\mathrm{p}=0.010^{*}$ \\
\hline ALT & $1.80(1.02,3.17)$ & $\mathrm{p}=0.041 *$ \\
\hline AST & $2.08(0.94,4.58)$ & $\mathrm{p}=0.070$ \\
\hline Total Bilirubin (mg/d) & $1.93(1.03,3.59)$ & $\mathrm{p}=0.039 *$ \\
\hline Total Cholesterol (mg/dL) & $3.46(1.27,9.40)$ & $\mathrm{p}=0.015^{*}$ \\
\hline High Density Lipoprotein (mg/dL) & $1.01(0.32,3.23)$ & $\mathrm{p}=0.981$ \\
\hline White Blood Cell Count & $2.31(1.01,5.28)$ & $\mathrm{p}=0.047 *$ \\
\hline Partial thromboplastin time & $1.65(0.73,3.75)$ & $\mathrm{p}=0.228$ \\
\hline
\end{tabular}




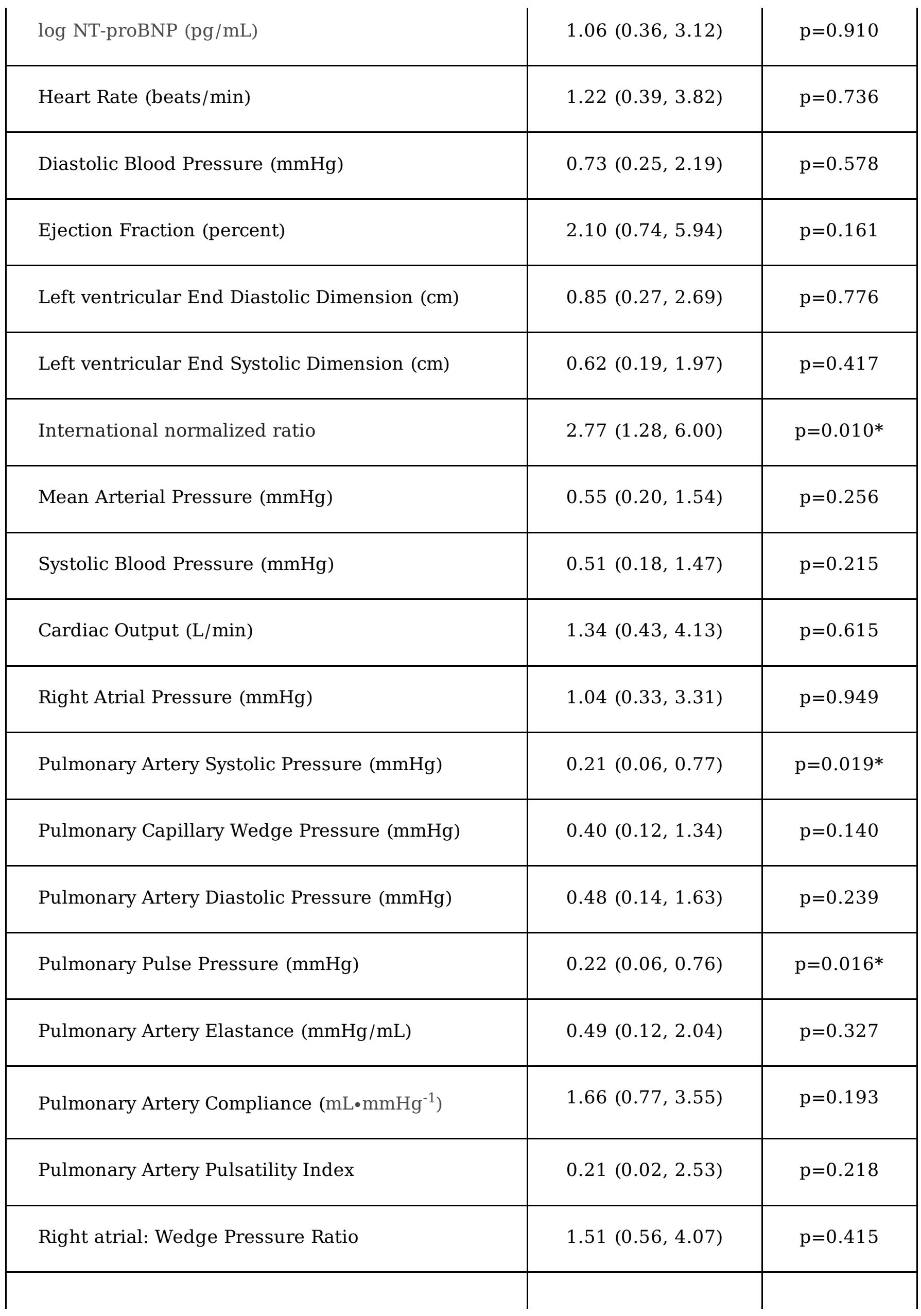




\begin{tabular}{|l|c|c|} 
Right Ventricular End Diastolic Pressure (mmHg) & $1.76(0.57,5.48)$ & $\mathrm{p}=0.327$ \\
\hline Systolic Blood Pressure (mmHg) & $0.51(0.18,1.47)$ & $\mathrm{p}=0.215$ \\
\hline Stroke Volume (mL) & $1.10(0.36,3.31)$ & $\mathrm{p}=0.870$ \\
\hline EUROMACS Score & $6.88(2.31,20.45)$ & $\mathrm{P}=0.001$ \\
\hline
\end{tabular}

Legend: Each odds ratio estimate reflects a 2 standard deviation change in the corresponding variable.

Table2b: Odds-ratios of each binary risk factor for early right heart failure based on Univariate Logistic Regression Models 


\begin{tabular}{|c|c|c|}
\hline Predictor & Odds-Ratio (95\% CI) & p-value \\
\hline Sex & $2.51(0.32,19.96)$ & $\mathrm{p}=0.384$ \\
\hline Bridge to Transplant LVAD & $0.68(0.21,2.2)$ & $\mathrm{p}=0.520$ \\
\hline Diabetes Mellitus & $0.51(0.15,1.73)$ & $\mathrm{p}=0.280$ \\
\hline Non-Ischemic Cardiomyopathy Type & $0.28(0.07,1.07)$ & $\mathrm{p}=0.063$ \\
\hline Coronary Artery Disease & $0.39(0.12,1.29)$ & $\mathrm{p}=0.123$ \\
\hline Atrial Fibrillation & $0.83(0.26,2.68)$ & $\mathrm{p}=0.755$ \\
\hline History of Coronary Artery Bypass Surgery & $0.77(0.16,3.62)$ & $\mathrm{p}=0.739$ \\
\hline Chronic Obstructive Pulmonary Disease & $0.62(0.13,2.91)$ & $\mathrm{p}=0.545$ \\
\hline Hypercholesterolemia & $1.14(0.36,3.64)$ & $\mathrm{p}=0.823$ \\
\hline Hypertension & $0.85(0.27,2.7)$ & $\mathrm{p}=0.779$ \\
\hline Implanted Cardiac Defibrillator & $0.55(0.16,1.89)$ & $\mathrm{p}=0.340$ \\
\hline History of Smoking & $0.85(0.18,4.02)$ & $\mathrm{p}=0.840$ \\
\hline Ventilator Use & $4.34(1.22,15.45)$ & $\mathrm{p}=0.023^{*}$ \\
\hline Continuous renal replacement therapy & $0(0, \operatorname{Inf})$ & $\mathrm{p}=0.992$ \\
\hline Inotropes & $2.76(0.73,10.45)$ & $\mathrm{p}=0.135$ \\
\hline Multiple Inotropes & $10.52(3.12,35.53)$ & $\mathrm{p}<0.001 *$ \\
\hline Extra corporal membrane oxygenation & $5.87(1.42,24.32)$ & $\mathrm{p}=0.015^{*}$ \\
\hline Impella & $10.91(0.92,129.67)$ & $\mathrm{p}=0.058$ \\
\hline
\end{tabular}


Table 2c: Likelihood ratio test p-value corresponding to each categorical risk factor for early right heart based on univariate logistic regression models. (Omitting odd-ratios as there are multiple per variable)

\begin{tabular}{|c|c|c|}
\hline \multicolumn{2}{|c|}{ Odds-Ratio (95\% CI) } & p-value \\
\hline Predictor & -- & $\mathrm{p}=0.523$ \\
\hline Race & -- & $\mathrm{p}=0.142$ \\
\hline INTERMACS Profile & -- & $\mathrm{p}=0.307$ \\
\hline Right Ventricle Function & -- & $\mathrm{p}=0.890$ \\
\hline Right Ventricle Size & -- & $\mathrm{p}=0.293$ \\
\hline Tricuspid Regurgitation Grade & - & \\
\hline
\end{tabular}

Legend INTERMACS: Interagency Registry for Mechanical Circulatory Support

\section{Figures}

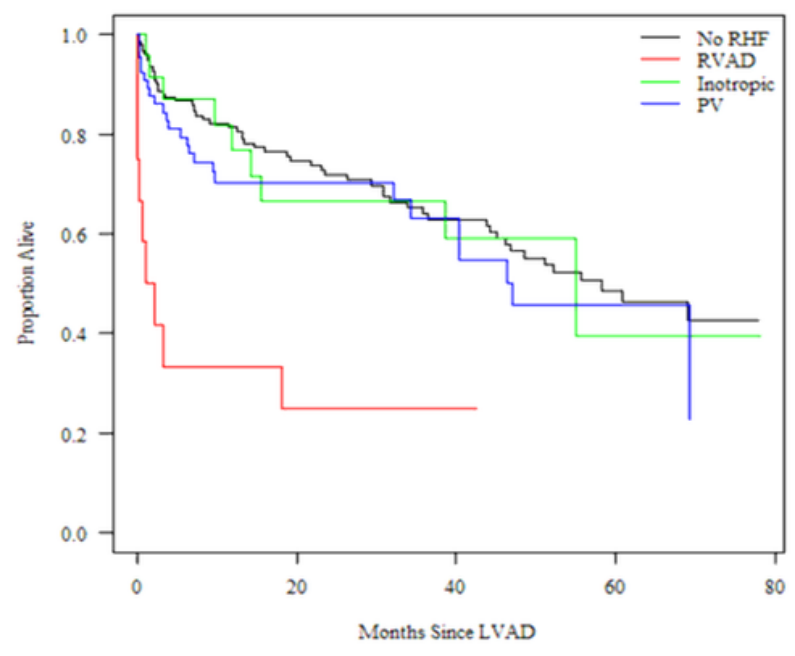


Figure 1

Kaplan Meier Survival Curves of LVAD Recipients by Type of Right Heart Failure Legend: RHF: Right Ventricular Heart Failure, RVAD: Right Ventricular Assist Device within 30 days, Inotropic: Inotrope Use $>14$ days after LVAD, PV:

Pulmonary Vasodilator Use $>48$ hours

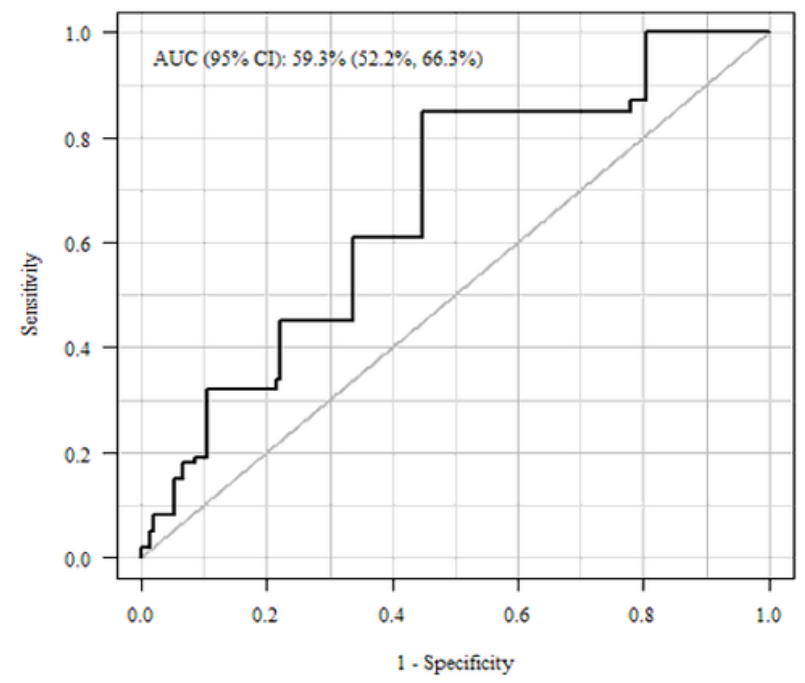

\section{Figure 2}

Receiver Operator Curve for the Performance of the EUROMACS Right Ventricular Risk Score in an External, Validation Cohort Legend: AUC: area under the curve, with $95 \%$ confidence interval. 